\title{
THUNDER piezoelectric actuators as a method of stretch-tuning an optical fiber grating
}

\author{
Sidney G. Allison, Robert L. Fox, Mark E. Froggatt and Brooks A. Childers \\ NASA Langley Research Center, Hampton, Virginia 23681-2199
}

\begin{abstract}
A method of stretching optical fiber holds interest for measuring strain in smart structures where the physical displacement may be used to tune optical fiber lasers. A small, light weight, low power tunable fiber laser is ideal for demodulating strain in optical fiber Bragg gratings attached to smart structures such as the re-usable launch vehicle that is being developed by NASA. A method is presented for stretching optical fibers using the THUNDER piezoelectric actuators invented at NASA Langley Research Center. THUNDER actuators use a piezoelectric layer bonded to a metal backing to enable the actuators to produce displacements larger than the unbonded piezoelectric material. The shift in reflected optical wavelength resulting from stretching the fiber Bragg grating is presented. Means of adapting THUNDER actuators for stretching optical fibers is discussed, including ferrules, ferrule clamp blocks, and plastic hinges made with stereo lithography.
\end{abstract}

Keywords: Tuning, optical, fiber Bragg grating, piezoelectric, actuator

\section{INTRODUCTION}

Optical fibers can be used to measure strain and other parameters in smart structures. ${ }^{1-8}$ Tunable lasers have become widely used as a means of measuring gratings in optical fibers because they provide a variable wavelength output. One method of generating a variable wavelength output is by stretching an optical fiber laser. This paper describes a method of stretching an optical fiber ${ }^{9}$ containing a Bragg grating to produce a shift in the reflected wavelength. This method holds promise because it may be used to tune an optical fiber laser in the same way it is shown herein to tune a grating.

The method described herein uses a piezoelectric device developed at NASA Langley Research Center. ${ }^{10}$ This device is known as Thin Layer Composite Unimorph Ferroelectric Driver and Sensor (THUNDER). As a driver, it produces physical displacement and/or force in response to a varying applied controlled voltage. For this application, two actuators are used to pull at both ends of an optical fiber containing a Bragg grating in order to produce a shift in the reflected wavelength. When an optical fiber is stretched, by a pulling force that is applied to both its ends, the Bragg grating's reflected light varies in spectral frequency and the variation in wavelength is a function of the force by which the ends of the optical fiber with grating are pulled apart.

\section{STRETCH-TUNING HARDWARE}

\subsection{Piezoelectric actuators}

Figure 1 shows the sandwich-like layup of THUNDER actuators for which test results are presented in this paper. The metal base layer is a sheet of 301 stainless steel $0.1016 \mathrm{~mm}$ thick. The piezoelectric wafer is a sheet of PZT-5A $0.2032 \mathrm{~mm}$ thick. The top layer is a piece of aluminum foil $0.0254 \mathrm{~mm}$ thick. An electrode layer consisting of a narrow strip of stainless steel foil is placed on each side of the piezoelectric layer and is in electrical contact with the PZT-5A layer in the finished actuator to provide a means of applying drive voltage. A $0.0254 \mathrm{~mm}$ thick layer of polyimid adhesive ${ }^{11-12}$ that cures at 325 degrees $\mathrm{C}$ is placed between the base metal layer and the PZT-5A and another layer is placed between the PZT$5 \mathrm{~A}$ and the aluminum top layer. 


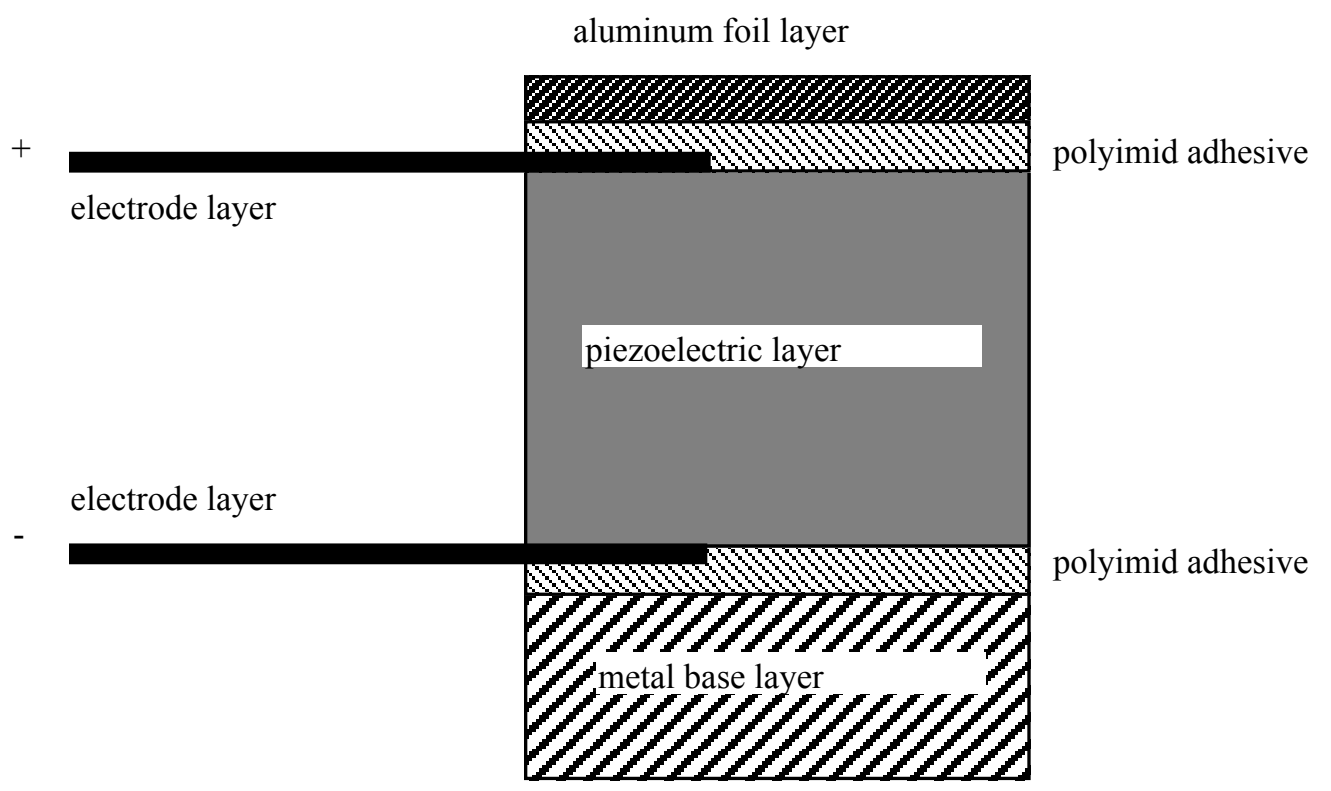

Figure 1 Typical cross-sectional layup of a THUNDER actuator. Electrodes are added to provide a means of applying drive voltage permitting generation of the necessary electric field to the PZT-5A piezoelectric layer. The layers are bonded together using a polyimid adhesive which cures at 325 degrees $\mathrm{C}$.

The PZT-5A sheet in Figure 1 is oriented to utilize the piezoelectric effect in the strip geometry ${ }^{13}$ shown in figure 2 . This geometry uses the $\mathrm{d}_{31}$ piezoelectric strain coefficient producing displacement in the 1-direction (lengthwise) as a result of electric field in the 3-direction (through the thickness). Since $\mathrm{d}_{31}$ is negative, strips always expand for a negative voltage applied as shown in Figure 1. The THUNDER actuators shown in Figure 2 were manufactured by laying up a metal base sheet, PZT-5A sheet and aluminum foil sheet, each having dimensions of $2.38 \mathrm{~cm}$ wide and $6.03 \mathrm{~cm}$ long. The sandwich layup is placed on a flat surface, bagged and cured for 30 minutes in an autoclave at 325 degrees C using $0.689 \mathrm{Mpa}$ pressure. The layers become bonded together at the cure temperature. When the layup is cooled to room temperature, it assumes a curved shape as shown in the actuator top view in Figure 2. The characteristic curvature seen in Figure $2 \mathrm{~b}$ comes about because the metal base plate has a larger coefficient of thermal expansion than does the PZT-5A and therefore shrinks more than does the PZT-5A when cooled. The aluminum foil top layer serves to make the actuators more rugged, while allowing the actuator to take the characteristic curved shape. An advantage of this manufacturing process which bonds the layers together at high temperature is that cooling to room temperature places the PZT-5A layer in a compressive prestressed condition which reduces the tendency for cracking. Actuators produced using this method have greatly improved output motion compared to that of the unbonded piezoelectric material.

Each actuator is polarized by applying 320 volts across the electrodes in the polarity direction shown in Figures 1 and 2 and holding for 2 minutes. Once poled, application of voltage in the same polarity causes the actuators to flatten (become less curved), resulting in an end-to-end displacement which can be used to stretch an optical fiber. These actuators offer advantages of being small and light weight. Another advantage is low power consumption required because this actuator design makes it a capacitive load. 


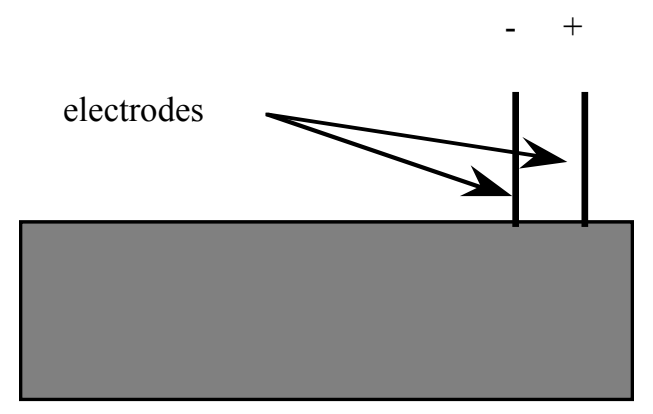

a) actuator side view

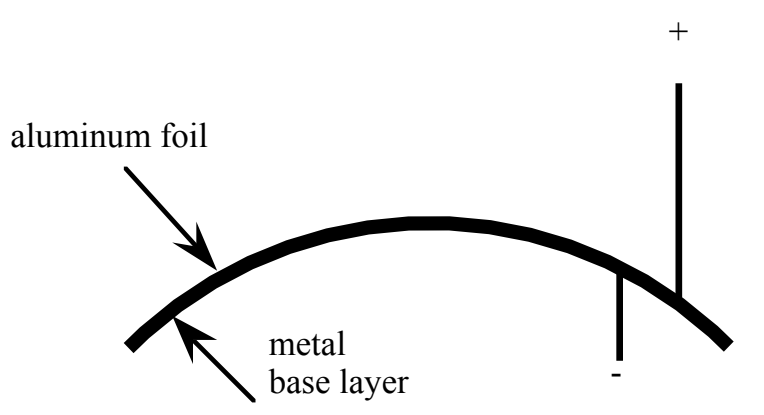

b) actuator top view

Figure 2 Side view and top view of a THUNDER actuator. After curing in an autoclave, cooling causes the actuator to take on a characteristic curved shape due to differential thermal contraction of the layers.

\subsection{Optical fiber with Bragg grating}

Figure 3 shows the optical fiber used in this experiment. The fiber is a single mode optical fiber and contains a Bragg grating written at 1550.2 nanometers. The fiber is coated with polyimid and has a $0.152 \mathrm{~mm}$ overall diameter including the coating. Stainless steel ferrules having $0.28 \mathrm{~mm}$ inside diameter and $0.36 \mathrm{~mm}$ outside diameter are bonded to the section of fiber on each side of the grating. The metal ferrules provide a rigid structure suitable for gripping by mechanical means. The ferrules were selected so as to allow a bond thickness of $0.076 \mathrm{~mm}$ to $0.127 \mathrm{~mm}$ which produces maximum bond strength for the adhesive used. The THUNDER actuators stretch the fiber, producing strain in the Bragg grating resulting in a shift in the reflected wavelength. The gauge length between the ferrules was chosen to be $4 \mathrm{~cm}$ because this is comparable to typical gauge length of erbium fiber lasers. Straining the Bragg grating in this fiber is expected to generate an optical wavelength shift similar to the shift that would be produced by straining the Bragg gratings and laser cavity in an erbium fiber laser if tested similarly. 


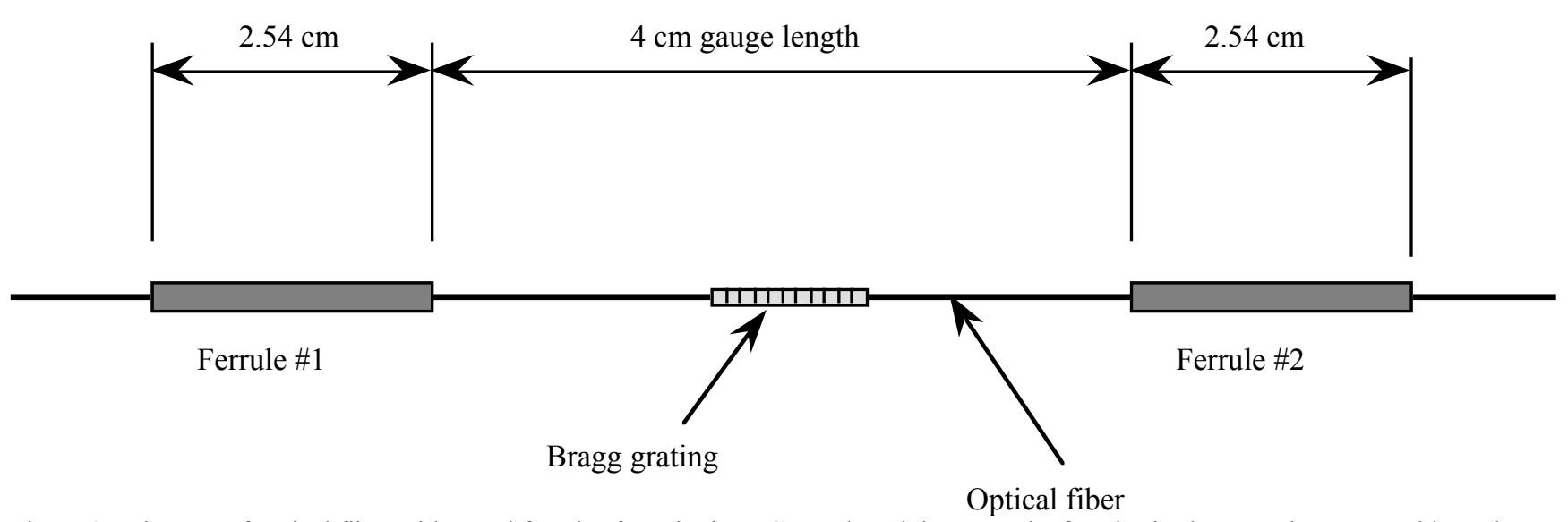

Figure 3 Diagram of optical fiber with metal ferrules for gripping. Gauge length between the ferrules is chosen to be comparable to that for a typical erbium fiber laser.

\subsection{Fiber stretcher hardware assembly}

Figure 4 is a diagram depicting the fiber stretcher hardware assembly used for tests in this paper. Two actuators were used together to increase stretching force and provide symmetry. Hinges for this stretcher are designed so that a pair of identical hinge-halves assemble together with a pin to form a hinge assembly to which a pair of THUNDER actuators attach. The ends of two THUNDER actuators fit into slots in the two hinge halves. This allows the actuator ends to rotate as the actuators flatten when driving voltage is applied, thereby producing a linear displacement at the hinge pin. Hinge-halves are designed so that each hinge assembly has room in the middle to fit an aluminum clamp block for transmitting hinge pin load to the fiber by clamping onto the ferrule. The hinge pin passes through the hinge halves and clamp block. The ferrule passes through the clamp block and hinge pin. The clamp block contains a set screw which is tightened to clamp onto and grip the ferrule thereby transmitting applied load from the actuator hinge pin to the fiber.

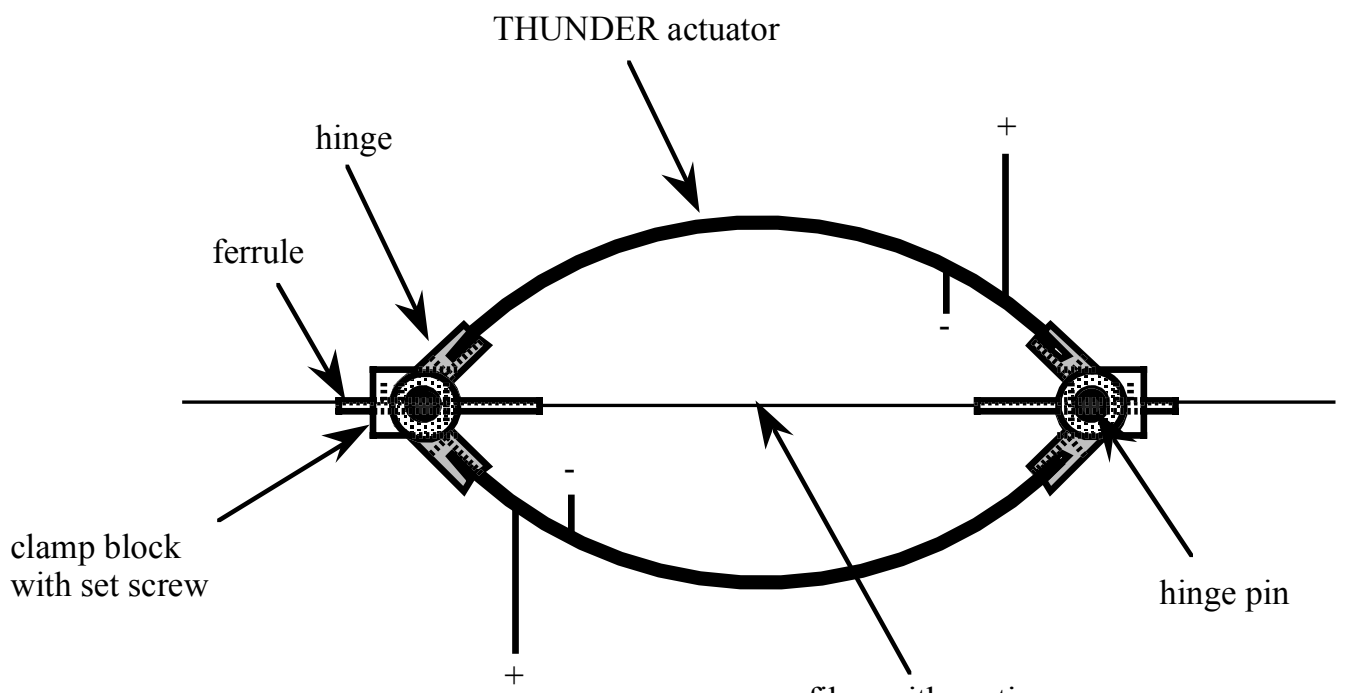

fiber with grating

Figure 4 Diagram showing top view of fiber stretcher hardware assembly. Actuators fit into slotted hinges. Hinge pins pass through clamp blocks. Ferrules pass through clamp blocks and hinge pins. A set screw in each clamp block grips each ferrule. 


\section{TEST SETUP}

\subsection{Test fixture}

Figure 5 is a diagram of the fiber stretcher displacement measurement apparatus. The apparatus consists of a metal base with a fixture at each end. The fixture shown on the left in Figure 5 is a holder for the fiber stretcher hardware assembly. It contains holes at the top and bottom into which fit the portions of the hinge pin extending beyond the fiber stretcher. This adapter prevents the left end of the stretcher from moving laterally while allowing the stretcher freedom to rotate about the pin. The fixture on the right holds a spring-loaded displacement probe. A displacement probe adapter attached to the hinge pin at the right end of the stretcher assembly produces a flat surface with which the spring-loaded displacement probe makes contact. This displacement probe adapter allows measurement of fiber stretcher displacement and contains a hole allowing clearance for the fiber and ferrule to pass through. Electrical leads for the two actuators are wired in parallel so that both actuators flatten when voltage is applied.

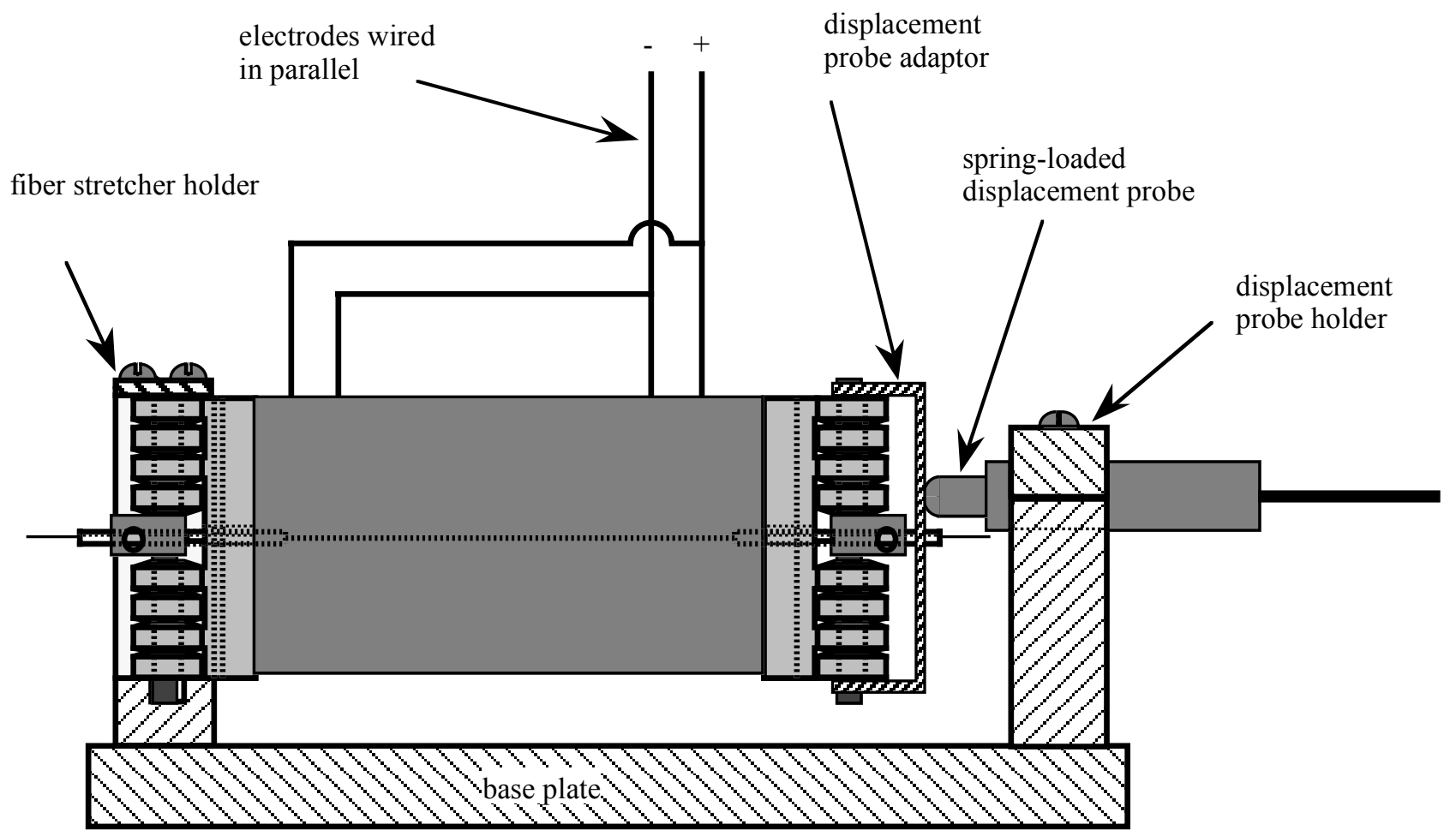

Figure 5 Diagram showing side view of fiber stretcher displacement measurement apparatus. Fixture at the left end of the base plate holds the fiber stretcher assembly. Fixture at the right end of the base plate holds the displacement probe. A displacement probe adapter attached to the hinge pin at the right side of the stretcher assembly provides a surface for the displacement probe to remain in contact with for measuring displacement during testing.

\subsection{Measurement system}

Figure 6 is a block diagram of the measurement system. The measurement system consists of a high voltage power supply, a multimeter used as a voltmeter, a readout system for the displacement transducer and an optical frequency domain reflectometer (OFDR). The high voltage power supply is a Kepco model BHK2000-0.1M with a voltage range of 0-2000 volts DC and accuracy of $1 \%$ of full scale. The voltmeter is a Hewlett Packard model 3478A multimeter having a range of $0-300$ volts DC and accuracy of $0.01 \%$ of the reading. Note that when performing measurements, voltage readings from 0 300 volts were taken from the multimeter and readings above 300 volts were taken from the voltmeter on the power supply. 
The displacement readout is a Federal Products Corp. model EAS1464 displacement measurement system having measurement range of $-1.016 \mathrm{~mm}$ to $1.016 \mathrm{~mm}$ and accuracy of $\pm 0.0127 \mathrm{~mm}$. The displacement probe is a Federal Products Corp. model EHE1102 displacement transducer having the same range and accuracy as the displacement readout. The $\mathrm{OFDR}^{3}$ consists of a tunable laser, a simple optical network, and a data acquisition system. The Bragg grating used in this work was produced during the fiber drawing process and has a low reflectivity estimated at $0.004 \%$. Typical Bragg grating readout systems require gratings with much higher reflectivites. However, the OFDR employs a coherent detection scheme and is capable of reading out very low reflectivity gratings. In fact, the OFDR is sensitive enough to measure strains by using Rayleigh scattering from the fiber core ${ }^{4}$ in lieu of a Bragg grating.

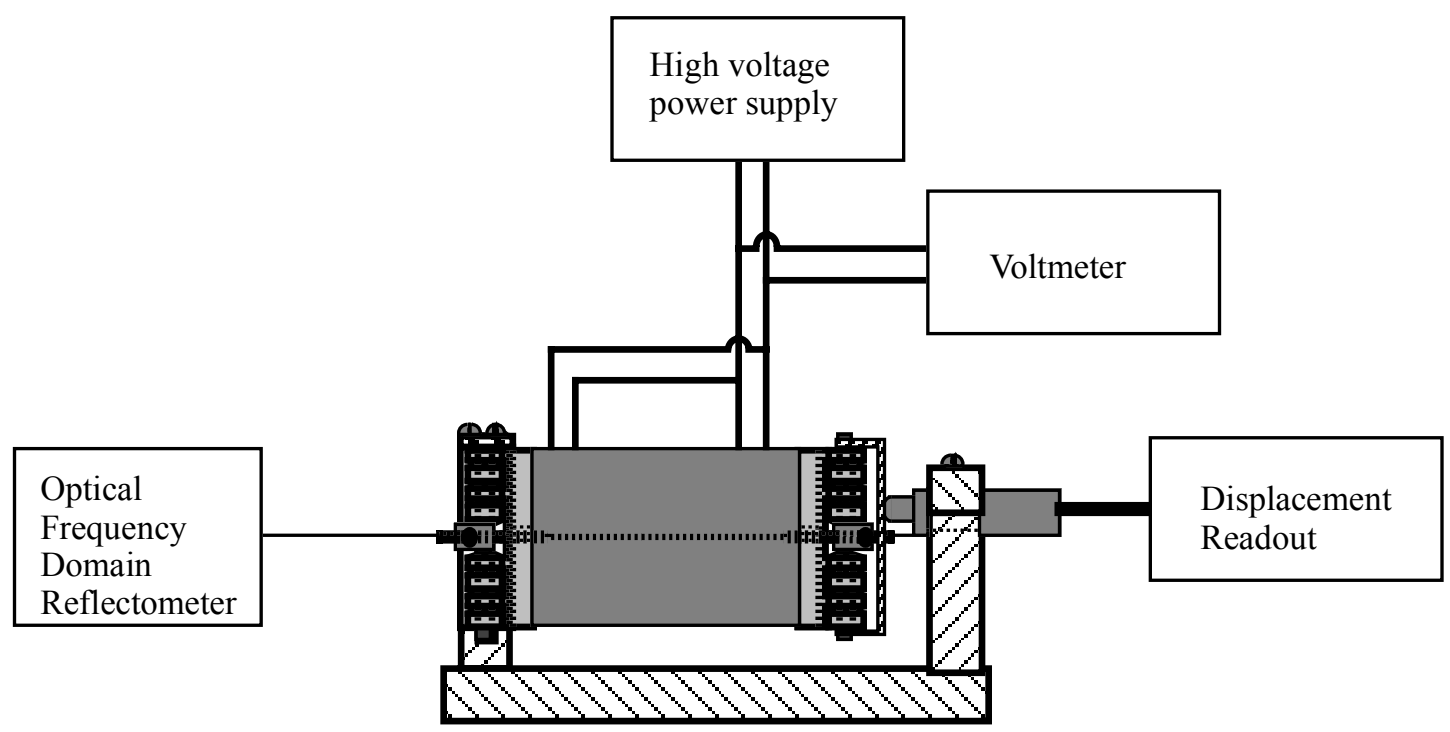

Figure 6 Block diagram of displacement and optical wavelength shift measurement system

\section{EXPERIMENTAL RESULTS}

\subsection{No-load displacement}

Figure 7 is a graph of displacement vs. voltage measured without stretching an optical fiber. Hysteresis seen in the figure is typical of THUNDER actuators. All piezoelectric ceramics exhibit hysteresis ${ }^{13}$ because of the difference in the strain that occurs when a particular voltage is approached from lower voltage vs. from higher voltage. Note the relatively large displacement of the THUNDER actuators. This graph helps demonstrate the improved output motion of the THUNDER actuators compared to the unbonded piezoelectric material. For comparison, we use piezoelectric theory ${ }^{13}$ to calculate the displacement of the ends of a unbonded $2.38 \mathrm{~cm} \times 6.03 \mathrm{~cm}$ strip of PZT-5A driven with the same 425 voltage signal level used in Figure 7:

where:

$$
\Delta \mathrm{L} / \mathrm{L}=\mathrm{d}_{31} \mathrm{~V} / \mathrm{t}
$$

$\mathrm{L}=$ length of PZT-5A strip $(6.03 \mathrm{~cm})$

$\Delta \mathrm{L}=$ displacement of ends of strip

$\mathrm{d}_{31}=$ piezoelectric strain coefficient $\left(171 \times 10^{-12} \mathrm{~m} / \mathrm{volt} / \mathrm{m}\right)$

$\mathrm{V}=$ driver voltage applied $(425 \mathrm{~V})$

$\mathrm{t}=$ thickness of PZT-5A strip $(0.2032 \mathrm{~mm})$.

This calculation indicates the unbonded PZT-5A strip would only produce $0.2157 \times 10^{-4} \mathrm{~m}$ of displacement of the ends compared to $4.8 \times 10^{-4} \mathrm{~m}$ for the actuators shown in Figure 7. However the reader should keep in mind that the increased displacement of the THUNDER actuators is gained at the expense of output force. 


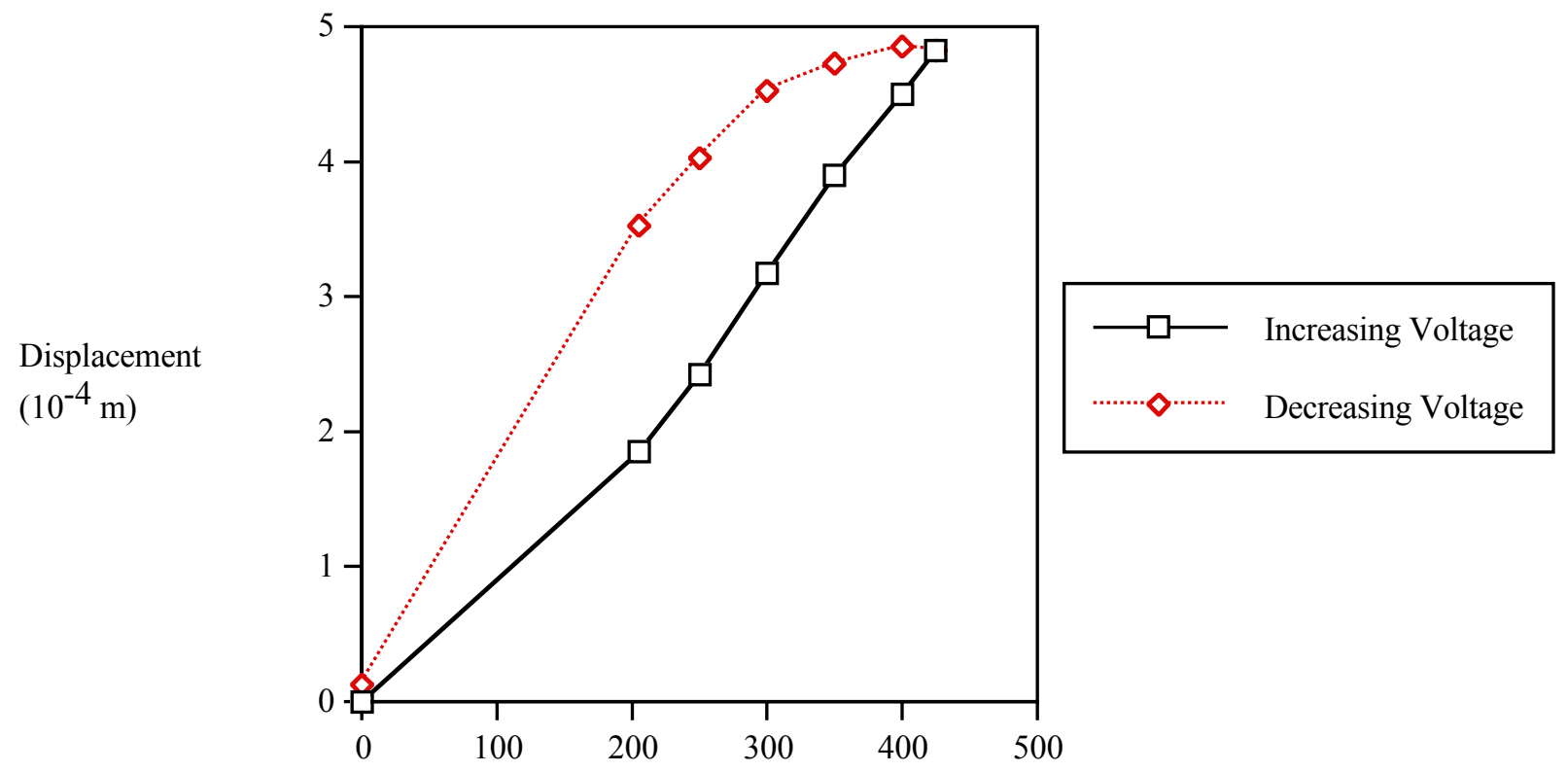

Volts

Figure 7 Graph of no-load displacement measurement results

\subsection{Wavelength shift and displacement}

Figure 8 is a graph of wavelength shift and displacement vs. voltage obtained while stretching a fiber with a Bragg grating. Typical measurement fiber applications require the tunable laser to produce 3-15 nm wavelength shift for gratings written at $1550 \mathrm{~nm}$. The $5.3 \mathrm{~nm}$ wavelength shift shown in Figure 8 is large enough for use with typical measurement fiber applications. Similar results are expected for an erbium fiber laser. 


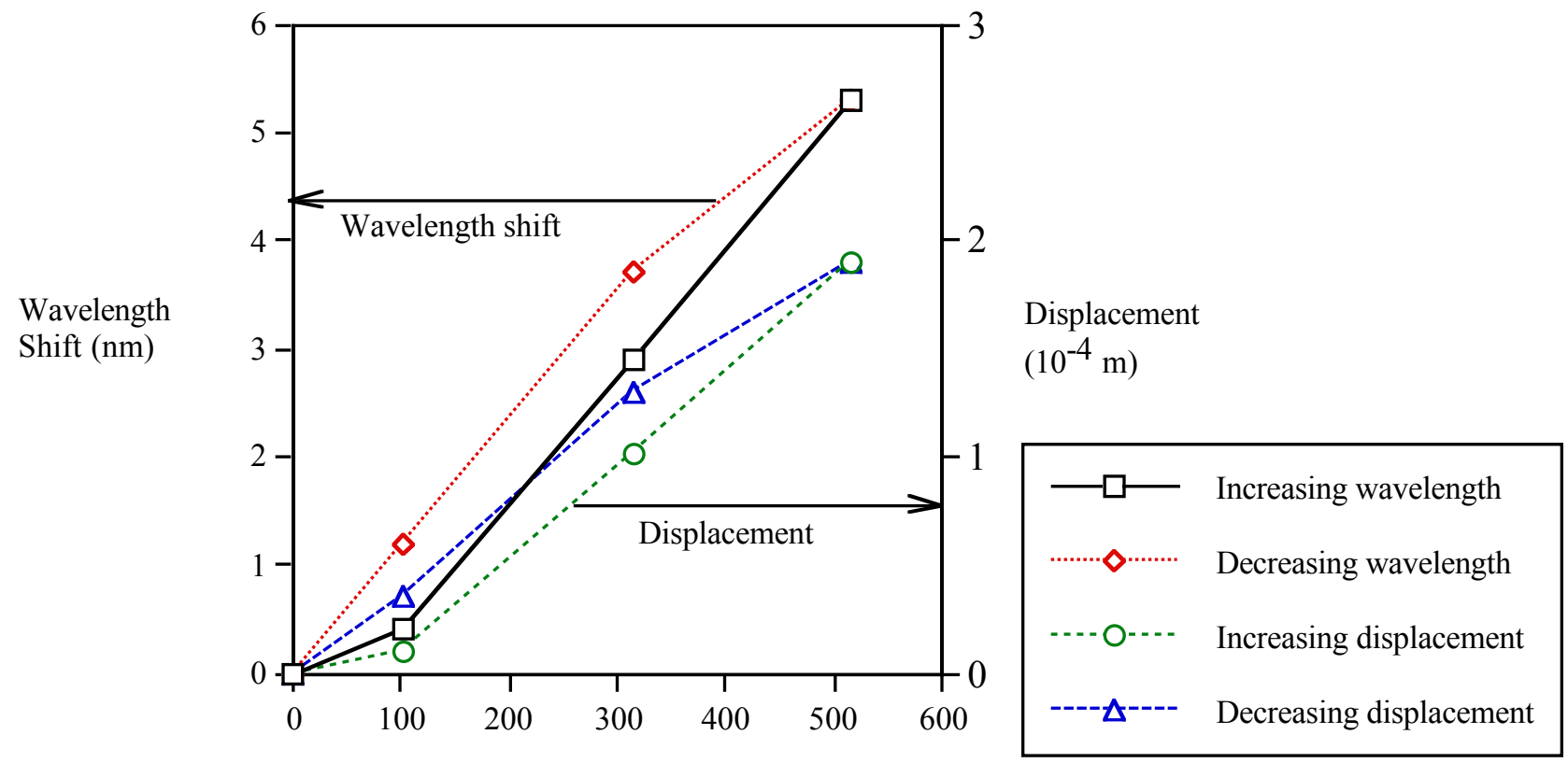

Volts

Figure 8 Graph of fiber Bragg grating wavelength shift and fiber stretcher displacement vs. applied voltage. Similar results are predicted should this fiber stretcher assembly be used to strain an erbium fiber laser.

In Figure 9, the same data presented in Figure 8 is re-plotted to compare normalized wavelength shift to fiber grating strain. A linear curve fit to the data points is shown along with the equation for the fit. This figure shows the effect of strain on the optical fiber grating. This measurement follows the well-known model $^{14}$ for Bragg grating wavelength shift:

$$
\Delta \lambda / \lambda=0.79 \varepsilon+\xi \Delta \mathrm{T}
$$

where $\Delta \lambda / \lambda$ is the normalized wavelength shift, $\varepsilon$ is strain in the fiber grating, $\xi$ is the thermo-optic coefficient and $\Delta \mathrm{T}$ is temperature change. Since the tests reported herein were done at room temperature, the thermal sensitivity term drops out of the equation, leaving only the strain term. The slope in the curve fit equation in Figure 9 is lower than in equation (2). This is most likely because a gauge length of $4 \mathrm{~cm}$ was assumed in calculating strain from the displacement data. The $4 \mathrm{~cm}$ length was used because that is the length of fiber between the ferrules. The actual gauge length is probably slightly longer because the ferrule gradually grips the fiber over some portion of the fiber that is in the fiber-to-ferrule bond line. 


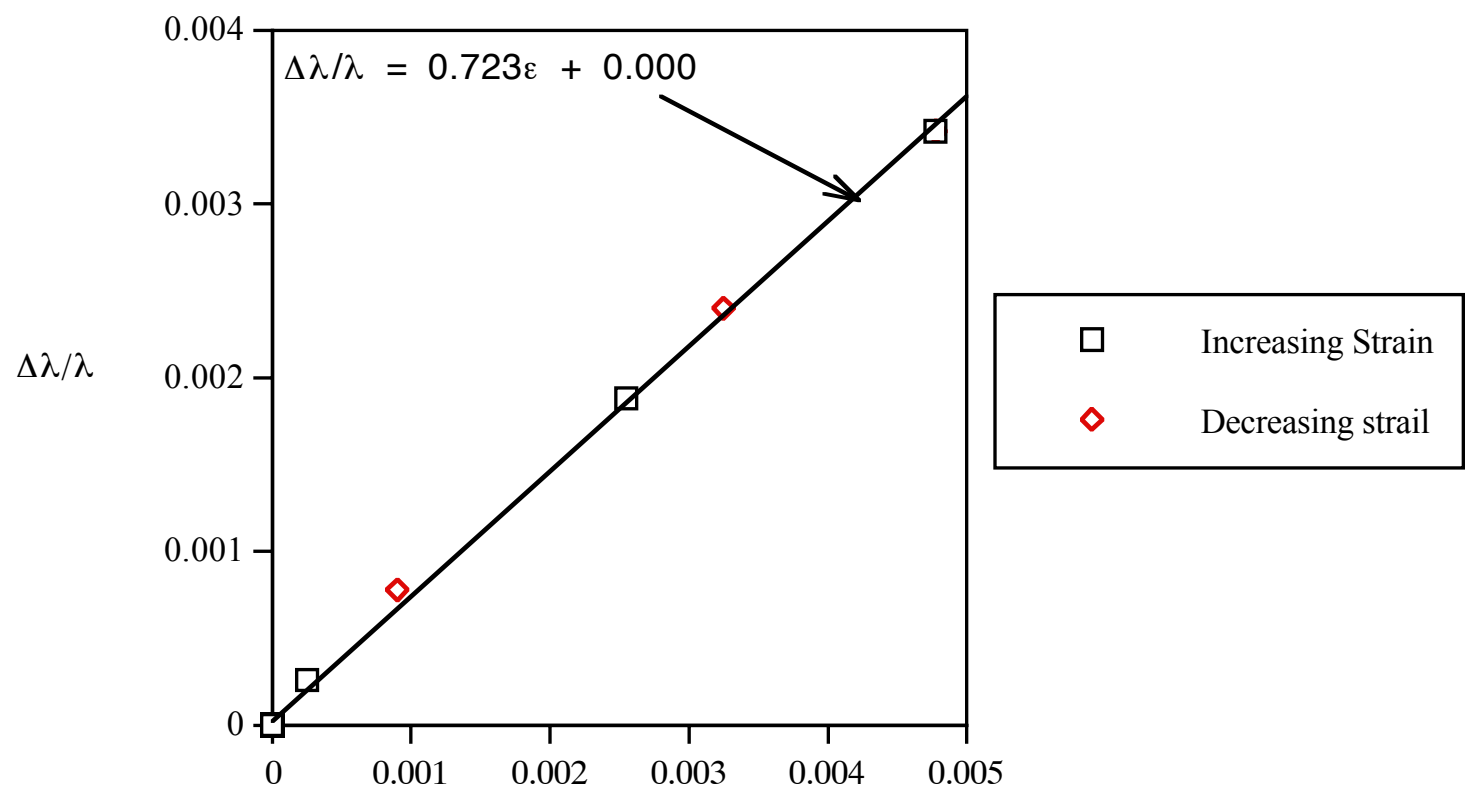

Strain $(\varepsilon)$

Figure 9 Graph of strain vs. normalized wavelength shift from the same data as in Figure 8. A curve fit is used to show the linear relationship between strain and normalized wavelength shift. The slope of the curve fit equation is comparable to that in the literature for the effect of strain on fiber gratings.

\section{CONCLUSIONS}

The THUNDER tunable laser has been shown to produce wavelength shifts large enough to demodulate Bragg gratings in measurement fibers such as those used in smart structures. Wavelength shifts this large are possible using piezoelectric material because layered construction enables the THUNDER actuators to produce much larger displacements than could be produced by the unbonded piezoelectric material. Measurement results show the expected relationship between normalized wavelength shift and strain. Similar behavior is expected for erbium fiber lasers. The technology lends itself well to being a practical solution for applications requiring small, light-weight, low power consumption tunable lasers.

\section{ACKNOWLEDGEMENTS}

The authors gratefully acknowledge the outstanding programmatic support provided by Leland D. Melvin. Melvin worked at the National Aeronautics and Space Administration as the X-33 Project Manager for Langley Research Center's Nondestructive Evaluation Sciences Branch.

\section{REFERENCES}

1. Moore, J. P., et al, "An Overview of the Fiber Optic Sensing System for Hydrogen Leak Detection in the Space Shuttle Discovery on STS-96", OSA Proceedings Bragg Gratings, Photosensitivity and Poling in Glass Waveguides, Stuart FL, Sep. 23-25, 1999.

2. Froggatt, M. E. and Bowen, W., "Optical Time Domain Reflectometry in Optical Fiber with Reflection Delay Time Matched to the Period of the Optical Frequency Modulation," Appl. Opt., Vol. 37, No. 10, pp. 1731-1734, 1 April 1998. 
3. Froggatt, M. E. and Moore, J., "Distributed Measurement of Static Strain in an Optical Fiber with Multiple Bragg Gratings at Nominally Equal Wavelengths," Appl. Opt., Vol. 37, No. 10, pp. 1741-1746, 1 April, 1998.

4. Froggatt, M. E. and Moore, J. "High Spatial-Resolution Distributed Strain Measurement in Optical Fiber with Rayleigh Scatter," Appl. Opt. , Vol. 37, No. 10, pp. 1735-1740, 1 April 1998.

5. Frogatt, M. E., U.S. patent 5,789,521 "Apparatus and Method for Measuring Strain in Bragg Gratings", 28 August 1998.

6. Brown, T., Wood, K., Childers, B., Cano, R., Jensen, B., and Rogowski, R.; "Fiber Optic Sensors for Health Monitoring of Morphing Aircraft", Proceedings of The International Society for Optical Engineering (SPIE), Vol. 3674, pp. 60-71, 1999.

7. Melvin, L. Childers, B., Rogowski, R., Prosser, W., Moore, J., Froggatt, M., Allison, S., Wu, M. C., Bly, J., Aude, C., Bouvier, C., Zisk, E., Enright, E., Cassadaban, Z., Reightler, R., Sirkis, J., Tang, I., Peng, T., Wegreich R., Garbos, R., Mouyos, W., Aibel, D., Bodan, P., "Integrated Vehicle Health Monitoring (IVHM) for Aerospace Vehicles," presented at the International Workshop on Structural Health Monitoring, Stanford, CA, September 18-20, 1997. In "Structural Health Monitoring Current Status and Perspectives", pp. 705-714, edited by Fu-Kuo Chang, Technomic Publishing Co. Inc., Lancaster, PA, ISBN No. 1-56676-605-2, 1997.

8. Froggatt, M. E. "Distributed Measurement of the Complex Modulation of a Photoinduced Bragg Grating in an Optical Fiber," Appl. Opt. , Vol. 35, No. 25, pp. 5162-5164, 1 September 1996.

9. Sidney G. Allison, Robert L. Fox, Antony Jalink Jr., Richard F. Hellbaum, Robert G. Bryant, Brooks A. Childers, Curtis R. Regan, Benjamin M. Copeland Jr., Walter L. Wade, James F. Linker, Daniel J. Carey, patent disclosure, NASA case number LAR 15776-1 "THUNDER Motor for Stretch-Tuning Optical Fiber Laser", filed February 25, 1998.

10. Hellbaum, Richard F., Bryant, Robert G., Fox, Robert L., U.S. patent 5,632,841 “Thin Layer Composite Unimorph Ferroelectric Driver and Sensor”, May 27, 1997.

11. Bryant, Robert G., U.S. patent 5741883 “Tough, soluble, aromatic, thermoplastic copolyimides”, April $21,1998$.

12. Bryant, Robert G., U.S. patent 5639850 "Process for preparing a tough, soluble, aromatic, thermoplastic copolyimide", 1998.

13. "IEEE Standard on Piezoelectricity”, ANSI/IEEE STD 176-1987, September 1987

14. "The Mechanical and Optical Reliability of Fiber Bragg Gratings," 3M Fiber Bragg Gratings Application Note, February 1996. 